TRANSACTIONS OF THE

AMERICAN MATHEMATICAL SOCIETY

Volume 350, Number 12, December 1998, Pages 5111-5122

S $0002-9947(98) 02088-1$

\title{
ON TRANSVERSALITY WITH DEFICIENCY AND A CONJECTURE OF SARD
}

\author{
CARLOS BIASI AND OSAMU SAEKI
}

\begin{abstract}
Let $f: M \rightarrow N$ be a $C^{r}$ map between $C^{r}$ manifolds $(r \geq 1)$ and $K$ a $C^{r}$ manifold. In this paper, by using the Sard theorem, we study the topological properties of the space of $C^{r}$ maps $g: K \rightarrow N$ which satisfy a certain transversality condition with respect to $f$ in a weak sense. As an application, by considering the case where $K$ is a point, we obtain some new results about the topological properties of $f\left(R_{q}(f)\right)$, where $R_{q}(f)$ is the set of points of $M$ where the rank of the differential of $f$ is less than or equal to $q$. In particular, we show a result about the topological dimension of $f\left(R_{q}(f)\right)$, which is closely related to a conjecture of Sard concerning the Hausdorff measure of $f\left(R_{q}(f)\right)$.
\end{abstract}

\section{INTRODUCTION}

Let $M, N$ and $K$ be $C^{r}$ manifolds of dimensions $m, n$ and $k$ respectively and $f: M \rightarrow N$ a $C^{r}$ map $(r \geq 1)$. For a nonnegative integer $l$, a $C^{r}$ map $g: K \rightarrow N$ is said to be transverse to $f$ with deficiency at most $l$ if for every pair $(x, y) \in K \times M$ such that $g(x)=f(y)$, we have

$$
\operatorname{dim}\left(d g_{x}\left(T_{x} K\right)+d f_{y}\left(T_{y} M\right)\right) \geq n-l .
$$

Let $T_{f}^{l}$ be the set of the $C^{r}$ maps $g: K \rightarrow N$ which are transverse to $f$ with deficiency at most $l$. Note that $T_{f}^{l}$ is a subset of the mapping space $C^{r}(K, N)$ equipped with the Whitney $C^{r}$ topology. In this paper, we study the topological properties of the space $T_{f}^{l}$ by using the Sard theorem [S1], [S2], [S3].

Our first result is that if

$$
r \geq \frac{m+k-n+l+1}{l+1},
$$

then the set $T_{f}^{l}$ is dense in $C^{r}(K, N)$ (Theorem 2.4). More specifically, it is residual. More generally, we show that, under the assumption that $K$ be compact, every continuous map $\varphi: P \rightarrow C^{r}(K, N)$ of a compact $p$-dimensional $C^{r}$ manifold $P$ can be approximated by a continuous map whose image is contained in $T_{f}^{l}$, provided that

$$
r \geq \frac{m+k-n+l+1}{l-p+1} \text { and } \quad p \leq l
$$

Received by the editors November 14, 1996

1991 Mathematics Subject Classification. Primary 57N75; Secondary 57R45, 55M10.

The second author has been partially supported by CNPq, Brazil, and by the Grant-in-Aid for Encouragement of Young Scientists (no. 08740057), Ministry of Education, Science and Culture, Japan.

(C)1998 American Mathematical Society 
(Theorem 2.9). As a corollary, we show that the inclusion $T_{f}^{l} \rightarrow C^{r}(K, N)$ induces an isomorphism $\pi_{i}\left(T_{f}^{l}\right) \rightarrow \pi_{i}\left(C^{r}(K, N)\right)$ for all $i$ with $i \leq p-1$ and an epimorphism for $i=p$ (Corollary 3.1).

Note that when $K$ is a point, $C^{r}(K, N)$ and $T_{f}^{l}$ are naturally identified with $N$ and $N-f\left(R_{q}(f)\right)$ respectively with $q=n-l-1$, where $R_{q}(f)(\subset M)$ is the set of points of $M$ where the rank of the differential of $f$ is less than or equal to $q$. Then using our result, we recover a result of Church on the homotopy property of the inclusion $N-f\left(R_{q}(f)\right) \rightarrow N$ [C2], [C3] as a corollary. Furthermore, we show that if $r \geq(m-q) /(s+1)$ for nonnegative integers $q$ and $s$, then the topological dimension of $f\left(R_{q}(f)\right)$ is less than or equal to $q+s$ (Corollary 3.6). This is a refinement of [S3, Theorem 2 (p.173)] and [C3, Proposition 4] (see also [C1]). Recall that Sard conjectures that $f\left(R_{q}(f)\right)$ is of $(q+\rho)$-dimensional measure zero if $r \geq(m-q) / \rho$ for $\rho>0$ (see [S3, $\S 5$, Statement 2]). If this conjecture is true, then the above result follows immediately. (Just consider $\rho=s+1$.) Since the Sard conjecture has not yet been proved, our result gives "supporting evidence" of the conjecture.

The paper is organized as follows. In $\S 2$, we recall the Sard theorem and use it to prove that the space $T_{f}^{l}$ is dense in $C^{r}(K, N)$ under some dimension assumptions. We also prove an approximation theorem for continuous maps $\varphi: P \rightarrow C^{r}(K, N)$ by maps whose image is contained in $T_{f}^{l}$. In $\S 3$, we use the approximation theorem to deduce some homotopical properties of the pair $\left(C^{r}(K, N), T_{f}^{l}\right)$. For example, we will show that if $K$ is compact and $r \geq m+k-n+3$, then $T_{f}^{2}\left(\subset C^{r}\left(K, \mathbf{R}^{n}\right)\right)$ is 1-connected.

Throughout the paper, all manifolds are assumed to have countable bases and may have boundary.

The second author would like to thank the people in ICMSC, University of São Paulo, for their hospitality and help during the preparation of the manuscript.

\section{SARD'S THEOREM AND ITS APPLICATION}

In the following, $M$ and $N$ will denote $m$ - and $n$-dimensional $C^{r}$ manifolds respectively, where $1 \leq r \leq \infty$. For a differentiable map $f: M \rightarrow N$ and an integer $q$ with $0 \leq q \leq \min \{m, n\}$, we set $R_{q}(f)=\left\{x \in M: \operatorname{rank} d f_{x} \leq q\right\}$. For a topological space $X, \operatorname{dim} X$ will always denote its topological dimension (see [HW]).

In [S1, p.889], Sard has proved the following.

Theorem 2.1. Let $f: M \rightarrow N$ be a $C^{r} \operatorname{map}(r \geq 1)$. If $q<n$ and $r \geq(m-q) /(n-$ $q)$, then $f\left(R_{q}(f)\right)$ is of $n$-dimensional measure zero; in particular, $\operatorname{dim} f\left(R_{q}(f)\right) \leq$ $n-1$.

In fact, Sard proves the above theorem when $M$ is an open set of $\mathbf{R}^{m}, N=\mathbf{R}^{n}$, and $m>n$. One can extend this result to the general case of a map between $C^{r}$ manifolds by using the arguments in [S3], provided that $m>n$. When $m \leq n$, Theorem 2.1 follows from the following proposition.

Proposition 2.2. Let $f: M \rightarrow N$ be a $C^{r} \operatorname{map}(r \geq 1)$. If $s \geq \max \{0, m-q-r\}$ for $q$ with $0 \leq q \leq \min \{m, n\}$, then $\operatorname{dim} f\left(R_{q}(f)\right) \leq q+s$.

Proof. Since $s \geq m-q-r$ and $r \geq 1$, we have $r \geq \max \{m-(q+s), 1\}$. Thus by $[\mathrm{S} 3, \S 5 \text {, Theorem } 2 \text { or Proposition } 4]^{1}$ we have $\operatorname{dim} f\left(R_{q+s}(f)\right) \leq q+s$. On

\footnotetext{
${ }^{1}$ Church [C3] assumes that the manifolds do not have boundary. However, his argument works also in the case where the manifolds have boundary.
} 
the other hand, since $s \geq 0$, we have $f\left(R_{q}(f)\right) \subset f\left(R_{q+s}(f)\right)$ and consequently $\operatorname{dim} f\left(R_{q}(f)\right) \leq \operatorname{dim} f\left(R_{q+s}(f)\right)$ (see [HW, Theorem III 1 (p.26)]). This completes the proof.

Let us prove Theorem 2.1 when $m \leq n$. Set $s=n-q-1$, which satisfies $s \geq 0$. Since $s \geq m-q-r$, we see that $\operatorname{dim} \bar{f}\left(R_{q}(f)\right) \leq q+s=n-1$ by Proposition 2.2 . This completes the proof of Theorem 2.1.

In the following, for $C^{r}$ manifolds $K$ and $N, C^{r}(K, N)$ will denote the space of all $C^{r}$ maps of $K$ into $N$ equipped with the strong Whitney $C^{r}$-topology (see [H, Chapter 2]).

Definition 2.3. Consider differentiable maps $f: M \rightarrow N$ and $g: K \rightarrow N$, where $M, N$ and $K$ are differentiable manifolds of dimensions $m, n$ and $k$ respectively. For an integer $l$ with $0 \leq l \leq n$ and a point $x \in K$, we say that $g$ is transverse to $f$ with deficiency at most $l$ at $x$ if for every point $y \in M$ with $f(y)=g(x)$, we have

$$
\operatorname{dim}\left(d f_{y}\left(T_{y} M\right)+d g_{x}\left(T_{x} K\right)\right) \geq n-l .
$$

For a subset $V$ of $K$, we say that $g$ is transverse to $f$ with deficiency at most $l$ on $V$ if $g$ is so at every point $x \in V$. We say that $g$ is transverse to $f$ with deficiency at most $l$ if $g$ is so on the whole of $K$. Note that when $l=0$, this notion is nothing but the usual notion of transversality.

For a differentiable map $f: M \rightarrow N$ and a nonnegative integer $l$, we denote by $T_{f}^{l}\left(\subset C^{r}(K, N)\right)$ the set of the $C^{r}$ maps which are transverse to $f$ with deficiency at most $l$.

As an application of Sard's theorem (Theorem 2.1), we show the following.

Theorem 2.4. Let $f: M \rightarrow N$ and $g: K \rightarrow N$ be $C^{r}$ maps, where $M, N$ and $K$ are $C^{r}$ manifolds of dimensions $m, n$ and $k$ respectively $(r \geq 1)$. If

$$
r \geq \frac{m+k-n+l+1}{l+1}
$$

for a nonnegative integer $l$, then there exists a $C^{r}$ map $h: K \rightarrow N$ homotopic to $g$ such that $h$ is transverse to $f$ with deficiency at most $l$. Furthermore, we can choose $h$ arbitrarily close to $g$ in the space $C^{r}(K, N)$. In particular, $T_{f}^{l}$ is dense in $C^{r}(K, N)$.

Compare the above theorem with [H, Theorem 2.7 (p.79)].

In the following, we use the notation as in [H, Chapter 2].

Lemma 2.5. Let $\left\{\varphi_{i}, U_{i}\right\}_{i \in \Lambda}$ be a locally finite set of charts on $K, C=\left\{C_{i}\right\}_{i \in \Lambda}$ a family of compact sets of $K$ with $C_{i} \subset U_{i}$, and $\varepsilon=\left\{\varepsilon_{i}\right\}_{i \in \Lambda}$ a family of positive numbers. Then there exists a $C^{r}$ function $\delta: K \rightarrow \mathbf{R}$ such that $\delta(x)>0$ for all $x \in K$ and $\left\|D^{k}\left(\delta \circ \varphi_{i}^{-1}\right)(x)\right\|<\varepsilon_{i}$ for all $x \in \varphi_{i}\left(C_{i}\right)$ and $k=0,1, \cdots, r$.

Proof. We may assume that $\left\{U_{i}\right\}_{i \in \Lambda}$ is a locally finite open covering of $K$ and that $\left\{C_{i}\right\}_{i \in \Lambda}$ is also a locally finite covering of $K$ (for this, construct such families first and then add the original families to them). Let $\left\{\lambda_{i}\right\}_{i \in \Lambda}$ be a $C^{r}$ partition of unity subordinate to $\left\{U_{i}\right\}_{i \in \Lambda}$ such that the support supp $\lambda_{i}$ of $\lambda_{i}$ is compact and that $\operatorname{supp} \lambda_{i} \supset C_{i}$. For each $i \in \Lambda$, set $\Lambda_{i}=\left\{j \in \Lambda: C_{i} \cap \operatorname{supp} \lambda_{j} \neq \emptyset\right\}$, which is a finite set whose cardinality we denote by $m_{i}$. Furthermore, set $\mu_{i}=\max \left\{\left\|D^{k}\left(\lambda_{j} \circ \varphi_{i}\right)(x)\right\|\right.$ : $\left.j \in \Lambda_{i}, x \in \varphi_{i}\left(C_{i}\right), k=0,1, \cdots, r\right\}$. Then we choose the family of positive numbers 
$\alpha=\left\{\alpha_{i}\right\}_{i \in \Lambda}$ so small that, putting $\beta_{i}=\max \left\{\alpha_{j}: j \in \Lambda_{i}\right\}$, we have $m_{i} \mu_{i} \beta_{i}<\varepsilon_{i}$. Then the $C^{r}$ function $\delta: K \rightarrow \mathbf{R}$ defined by

$$
\delta(x)=\sum_{i \in \Lambda} \alpha_{i} \lambda_{i}(x)
$$

for $x \in K$ satisfies the required conditions. This completes the proof.

Proof of Theorem 2.4. We may assume that $N$ is a $C^{\infty}$ manifold (for example, see [H, Theorem 2.9 (p.51)]). By Whitney's embedding theorem [W], we have a proper ${ }^{2}$ $C^{\infty}$ embedding $i: N \rightarrow \mathbf{R}^{2 n+1}$. We identify $N$ with $i(N)$. Let $V(N)$ be an open tubular neighborhood of $N$ in $\mathbf{R}^{2 n+1}$ and $\pi: V(N) \rightarrow N$ the natural projection, which is a $C^{\infty}$ map. Note that $\pi$ is a submersion.

Let $\mathcal{U}$ be an arbitrary open neighborhood of $g$ in $C^{r}(K, N)$. Since

$$
\pi_{*}: C^{r}(K, V(N)) \rightarrow C^{r}(K, N)
$$

is a continuous map, $\pi_{*}^{-1}(\mathcal{U})$ is an open neighborhood of $i \circ g$ in $C^{r}(K, V(N))$. Then there exist $\Phi=\left\{\varphi_{i}, U_{i}\right\}_{i \in \Lambda}$ (a locally finite set of charts on $K$ ), $C=\left\{C_{i}\right\}_{i \in \Lambda}$ (a family of compact sets of $K$ with $C_{i} \subset U_{i}$ ), and $\varepsilon=\left\{\varepsilon_{i}\right\}_{i \in \Lambda}$ (a family of positive numbers) such that $\mathcal{N}^{r}(i \circ g ; \Phi, C, \varepsilon)$ is contained in $\pi_{*}^{-1}(\mathcal{U})$, where $\mathcal{N}^{r}(i \circ g ; \Phi, C, \varepsilon)$ is the neighborhood of $i \circ g$ in $C^{r}(K, V(N))$ defined as in [H, p.35] using the obvious coordinate of $V(N)$ induced by that of $\mathbf{R}^{2 n+1}$. We may assume that $\left\{U_{i}\right\}_{i \in \Lambda}$ is a locally finite open covering of $K$ and that $\left\{C_{i}\right\}_{i \in \Lambda}$ is also a locally finite covering of $K$. Choosing $\varepsilon_{i}$ smaller if necessary, we may assume that $x+v \in V(N)$ for all $x \in g\left(C_{i}\right) \subset N$ and for all $v \in \mathbf{R}^{2 n+1}$ with $\|v\|<\varepsilon_{i}$, where $\mathbf{R}^{2 n+1}$ is equipped with the usual inner product and the norm. Let $\delta: K \rightarrow \mathbf{R}$ be a $C^{r}$ function as in Lemma 2.5 and let $\left\{X_{1}, \cdots, X_{2 n+1}\right\}$ be a fixed orthonormal basis of $\mathbf{R}^{2 n+1}$. Then define the map $G: \operatorname{Int} D^{s} \times K \rightarrow N$ by

$$
G\left(\lambda_{1}, \cdots, \lambda_{2 n+1} ; x\right)=\pi\left(g(x)+\delta(x) \sum_{j=1}^{2 n+1} \lambda_{j} X_{j}\right)
$$

for $\left(\lambda_{1}, \cdots, \lambda_{2 n+1}\right) \in \operatorname{Int} D^{2 n+1}$ and $x \in K$, where $\operatorname{Int} D^{2 n+1}=\left\{\left(\lambda_{1}, \cdots, \lambda_{2 n+1}\right)\right.$ : $\left.\sum_{j=1}^{2 n+1} \lambda_{j}^{2}<1\right\}$. Note that $G$ is a well-defined $C^{r}$ map and that $G$ is transverse to $f$ in the usual sense, since $G$ is a submersion ${ }^{3}$. For $\lambda=\left(\lambda_{1}, \cdots, \lambda_{2 n+1}\right) \in \operatorname{Int} D^{2 n+1}$, define $\tilde{g}_{\lambda}: K \rightarrow V(N)$ by

$$
\tilde{g}_{\lambda}(x)=g(x)+\delta(x) \sum_{j=1}^{2 n+1} \lambda_{j} X_{j}
$$

$(x \in K)$. Then by the above construction, we see that $\tilde{g}_{\lambda} \in \pi_{*}^{-1}(\mathcal{U})$ for all $\lambda \in$ $\operatorname{Int} D^{2 n+1}$ and hence that $g_{\lambda}=\pi \circ \tilde{g}_{\lambda} \in \mathcal{U}$.

Thus in order to prove the theorem, we have only to show that $g_{\lambda}$ is transverse to $f$ with deficiency at most $l$ for some $\lambda \in \operatorname{Int} D^{2 n+1}$. In the following, we set $s=2 n+1$.

Set $Z=\left\{(\lambda, x, y) \in \operatorname{Int} D^{s} \times K \times M: G(\lambda, x)=f(y)\right\}$, which is a $C^{r}$ manifold of dimension $m+k+s-n$, since $G$ is transverse to $f$ in the usual sense. Let $\pi: Z \rightarrow \operatorname{Int} D^{s}$ be the projection to the first factor $\operatorname{Int} D^{s} \times K \times M \rightarrow \operatorname{Int} D^{s}$ restricted to $Z$. Note that $\pi$ is a $C^{r}$ map.

\footnotetext{
${ }^{2} \mathrm{~A}$ continuous map is said to be proper if the inverse image of every compact set is compact.

${ }^{3}$ For the construction of such a map, see also [DK, §4.3.1].
} 
Lemma 2.6. We have

$$
s-\operatorname{rank} d \pi_{(\lambda, x, y)}=n-\operatorname{rank}\left(d\left(g_{\lambda}\right)_{x}+d f_{y}\right)
$$

for all $(\lambda, x, y) \in Z$.

Proof. By the definitions of $Z$ and $\pi$, we have

$$
T_{(\lambda, x, y)} Z=\left\{(v, w) \in T_{(\lambda, x)}\left(\operatorname{Int} D^{s} \times K\right) \times T_{y} M: d G_{(\lambda, x)}(v)=d f_{y}(w)\right\}
$$

and

$$
d \pi_{(\lambda, x, y)}\left(T_{(\lambda, x, y)} Z\right)=\left\{u \in T_{\lambda}\left(\operatorname{Int} D^{s}\right): d G_{(\lambda, x)}\left(u, u^{\prime}\right)=d f_{y}(w)\right.
$$

for some $u^{\prime} \in T_{x} K$ and $\left.w \in T_{y} M\right\}$.

Set $\xi=\left.d G_{(\lambda, x)}\right|_{T_{\lambda}\left(\operatorname{Int} D^{s}\right) \times\{0\}}: T_{\lambda}\left(\operatorname{Int} D^{s}\right) \rightarrow T_{z} N$, where $z=G(\lambda, x)=f(y)$. Then we have

$$
\begin{aligned}
d \pi_{(\lambda, x, y)}\left(T_{(\lambda, x, y)} Z\right) & =\left\{u \in T_{\lambda}\left(\operatorname{Int} D^{s}\right): \xi(u) \in d\left(g_{\lambda}\right)_{x}\left(T_{x} K\right)+d f_{y}\left(T_{y} M\right)\right\} \\
& =\xi^{-1}\left(d\left(g_{\lambda}\right)_{x}\left(T_{x} K\right)+d f_{y}\left(T_{y} M\right)\right) .
\end{aligned}
$$

Thus the linear map $\xi$ induces an injective linear map

$$
\bar{\xi}: T_{\lambda}\left(\operatorname{Int} D^{s}\right) / d \pi_{(\lambda, x, y)}\left(T_{(\lambda, x, y)} Z\right) \rightarrow T_{z} N /\left(d\left(g_{\lambda}\right)_{x}\left(T_{x} K\right)+d f_{y}\left(T_{y} M\right)\right) .
$$

Since $G$ is transverse to $f$ in the usual sense, we have

$$
\xi\left(T_{\lambda}\left(\operatorname{Int} D^{s}\right)\right)+d\left(g_{\lambda}\right)_{x}\left(T_{x} K\right)+d f_{y}\left(T_{y} M\right)=T_{z} N,
$$

which implies that $\bar{\xi}$ is surjective. Thus we have the conclusion, comparing the dimensions of the source and the target spaces of $\bar{\xi}$. This completes the proof of Lemma 2.6.

By our assumption, we have

$$
r \geq \frac{m+k-n+l+1}{l+1}=\frac{(m+k-n+s)-(s-l-1)}{s-(s-l-1)} .
$$

Thus, applying Theorem 2.1 to the $C^{r}$ map $\pi: Z \rightarrow \operatorname{Int} D^{s}$ with $q=s-l-1$, we have

$$
\operatorname{dim} \pi\left(R_{s-l-1}(\pi)\right) \leq s-1 .
$$

In particular, there exists a $\lambda_{0} \in \operatorname{Int} D^{s}$ such that $\lambda_{0} \notin \pi\left(R_{s-l-1}(\pi)\right)$. For this $\lambda_{0}$, we have $\operatorname{rank} d \pi_{\left(\lambda_{0}, x, y\right)} \geq s-l$ for all $(x, y) \in K \times M$ such that $\left(\lambda_{0}, x, y\right) \in$ $Z$. In particular, we have $s-\operatorname{rank} d \pi_{(\lambda, x, y)} \leq l$. Then by Lemma 2.6, we have $\operatorname{dim}\left(d\left(g_{\lambda_{0}}\right)_{x}\left(T_{x} K\right)+d f_{y}\left(T_{y} M\right)\right) \geq n-l$. Thus the $C^{r}$ map $h=g_{\lambda_{0}}$ is the required map. This completes the proof of Theorem 2.4.

Remark 2.7. It is not difficult to show that $T_{f}^{l}$ is an open set of $C^{r}(K, N)$, provided that $f$ is proper. We can prove this fact by using an argument similar to [H, Chapter 3]. (Note that a continuous map between manifolds is proper if and only if it is a closed map.) Thus, if

$$
r \geq \frac{m+k-n+l+1}{l+1}
$$

and $f$ is proper, then $T_{f}^{l}$ is an open dense subset of $C^{r}(K, N)$. When $f$ is not proper, $T_{f}^{l}$ is the intersection of a countable family of open dense subsets of $C^{r}(K, N)$; i.e., $T_{f}^{l}$ is residual (see [GG, Chapter II, §3], for example). We do not know if the assumption (2.8) is essential or not. 
We recall that when $K$ is compact, the mapping space $C^{r}(K, N)$ has the structure of a complete metric space (see [H, Theorem 4.4 (p.62)]). We denote by $d(g, h)$ the distance between two elements $g$ and $h$ of $C^{r}(K, N)$.

Theorem 2.9. Let $M, N, K$ and $P$ be $C^{r}$ manifolds of dimensions $m, n, k$ and $p$ respectively and let $f: M \rightarrow N$ be a (not necessarily proper) $C^{r} \operatorname{map}(r \geq 1)$. We suppose that $K$ and $P$ are compact. Furthermore, let $\mathcal{V}$ be an open subset of $C^{r}(K, N)$ which contains $T_{f}^{l}$ and let $\varphi: P \rightarrow \mathcal{V}$ be a continuous map such that $\varphi(F) \subset T_{f}^{l}$ for a closed subset $F$ of $P$. If

$$
r \geq \frac{m+k-n+l+1}{l-p+1} \text { and } \quad p \leq l
$$

for a nonnegative integer $l$, then for a given positive number $\varepsilon$, there exists a continuous map $\psi: P \rightarrow \mathcal{V}$ such that $\left.\psi\right|_{F}=\left.\varphi\right|_{F}, \psi$ is homotopic to $\varphi$ fixing $F$, $\psi(P) \subset T_{f}^{l}$, and $d(\psi(\lambda), \varphi(\lambda))<\varepsilon$ for all $\lambda \in P$.

Proof. First we consider the case where $f$ is proper and hence $T_{f}^{l}$ is open in $C^{r}(K, N)$. Then we may assume that $F$ is a codimension 0 submanifold of $P$. Furthermore, we may assume that there exists another codimension 0 submanifold $F^{\prime}$ of $P$ containing $F$ such that $\varphi\left(F^{\prime}\right) \subset T_{f}^{l}$ and that $F^{\prime}-\operatorname{Int} F$ is diffeomorphic to $\partial F \times[0,1]$.

Define the continuous map $\Phi: P \times K \rightarrow N$ by $\Phi(\lambda, x)=(\varphi(\lambda))(x)(\lambda \in P, x \in$ $K)$. Then there exists a $C^{r}$ map $\tilde{\Phi}: P \times K \rightarrow N$ such that $d(\tilde{\varphi}(\lambda), \varphi(\lambda))<\varepsilon / 2$ for all $\lambda \in P$ and $\tilde{\varphi}(P) \subset \mathcal{V}$, where $\tilde{\varphi}: P \rightarrow C^{r}(K, N)$ is the continuous map ${ }^{4}$ defined by $(\tilde{\varphi}(\lambda))(x)=\tilde{\Phi}(\lambda, x)$. Such a $C^{r}$ map can be constructed by an argument using the technique of integration (for example, see $[\mathrm{H}$, Chapter 2, §2] or the proof of $[\mathrm{M}$, Lemma 1.6]).

Since

$$
r \geq \frac{m+(p+k)-n+(l-p)+1}{(l-p)+1} \quad \text { and } \quad l-p \geq 0
$$

by our assumptions, Theorem 2.4 implies that there exists a $C^{r}$ map $\Psi^{\prime}: P \times K \rightarrow$ $N$ arbitrarily close to $\tilde{\Phi}$ in $C^{r}(P \times K, N)$ such that $\Psi^{\prime}$ is transverse to $f$ with deficiency at most $l-p$. Define the continuous map $\psi^{\prime}: P \rightarrow C^{r}(K, N)$ by $\left(\psi^{\prime}(\lambda)\right)(x)=\Psi^{\prime}(\lambda, x)(\lambda \in P, x \in K)$. Then we see easily that $\psi^{\prime}(x) \in T_{f}^{l}$ for all $x \in P$. Furthermore, we may assume that $d\left(\tilde{\varphi}(\lambda), \psi^{\prime}(\lambda)\right)<\varepsilon / 2$ and hence $d\left(\varphi(\lambda), \psi^{\prime}(\lambda)\right)<\varepsilon$ for all $\lambda \in P$.

As in the proof of Theorem 2.4, we may assume that $N$ is a closed $C^{\infty}$ submanifold of $\mathbf{R}^{2 n+1}$ and let $V(N)$ and $\pi: V(N) \rightarrow N$ be an open tubular neighborhood of $N$ in $\mathbf{R}^{2 n+1}$ and a natural projection map respectively. For $t \in[0,1]$ and $\lambda \in P$, define $\bar{h}_{t}(\lambda) \in C^{r}\left(K, \mathbf{R}^{2 n+1}\right)$ by $\left(\bar{h}_{t}(\lambda)\right)(x)=(1-t)(\varphi(\lambda))(x)+t\left(\psi^{\prime}(\lambda)\right)(x)(x \in K)$. Choosing $\varepsilon$ sufficiently small, we may assume that $\bar{h}_{t}(\lambda) \in C^{r}(K, V(N))$. Then define $h_{t}(\lambda) \in C^{r}(K, N)$ by $h_{t}(\lambda)=\pi \circ\left(\bar{h}_{t}(\lambda)\right)$. Choosing $\psi^{\prime}$ sufficiently close to $\varphi$, we may further assume that $h_{t}(\lambda) \in \mathcal{V}, h_{t}\left(F^{\prime}\right) \subset T_{f}^{l}$ and $d\left(h_{t}(\lambda), \varphi(\lambda)\right)<\varepsilon$ for all $t \in[0,1]$ and all $\lambda \in P$. Define the continuous map $H: P \times[0,1] \rightarrow \mathcal{V}$ by $H(\lambda, t)=h_{t}(\lambda)$. Note that $H$ is a homotopy between $\varphi$ and $\psi^{\prime}$. Fix a $C^{r}$ diffeomorphism $\eta: F^{\prime}-\operatorname{Int} F \rightarrow \partial F \times[0,1]$ with $\eta(w)=(w, 0)$ for all $w \in \partial F$ and

\footnotetext{
${ }^{4}$ In general, even if a map $\Theta: P \times K \rightarrow N$ is of class $C^{r}$, the associated map $\theta: P \rightarrow C^{r}(K, N)$ defined by $(\theta(\lambda))(x)=\Theta(\lambda, x)(\lambda \in P, x \in K)$ is not necessarily continuous. However, if $K$ is compact, then $\theta$ is always continuous.
} 
let $p_{2}: \partial F \times[0,1] \rightarrow[0,1]$ be the projection to the second factor. Then define the $\operatorname{map} \tilde{H}: P \times[0,1] \rightarrow \mathcal{V}$ by

$$
\tilde{H}(w, t)= \begin{cases}H(w, t) & \left({ }^{\forall} w \in P-\operatorname{Int} F^{\prime},{ }^{\forall} t \in[0,1]\right), \\ \varphi(w) & \left({ }^{\forall} w \in F,{ }^{\forall} t \in[0,1]\right), \\ H\left(x,\left(p_{2} \circ \eta(w)\right) t\right) & \left({ }^{\forall} w \in F^{\prime}-\operatorname{Int} F,{ }^{\forall} t \in[0,1]\right) .\end{cases}
$$

Then $\tilde{H}$ is a continuous map and $\tilde{H}(w, 0)=\varphi(w)$ for all $w \in P$. Define $\psi: P \rightarrow \mathcal{V}$ by $\psi(w)=\tilde{H}(w, 1)(w \in P)$. Then we see that $\psi$ satisfies all the required conditions. This completes the proof of Theorem 2.9 when $f$ is proper.

Now consider the general case where $f$ may not be proper. Since $P$ is compact, we see that there exists an open set $\mathcal{V}^{\prime}$ of $C^{r}(K, N)$ such that $\varphi(P) \subset \mathcal{V}^{\prime} \subset \overline{\mathcal{V}^{\prime}} \subset \mathcal{V}$. On the other hand, there exists a countable family of codimension 0 compact $C^{r}$ submanifolds $M_{1} \subset M_{2} \subset \cdots \subset M_{i} \subset M_{i+1} \subset \cdots$ of $M$ such that $\bigcup_{i=1}^{\infty} M_{i}=M$. Note that $\left.f\right|_{M_{i}}$ is a proper map and that

$$
T_{f}^{l}=\bigcap_{i=1}^{\infty} T_{\left(f \mid M_{i}\right)}^{l} .
$$

Then by the above argument, there exists a continuous map $\psi_{1}: P \rightarrow \mathcal{V}^{\prime}$ such that $\left.\psi_{1}\right|_{F}=\left.\varphi\right|_{F}, \psi_{1}(P) \subset T_{\left(\left.f\right|_{M_{1}}\right)}^{l}$ and $d\left(\varphi(\lambda), \psi_{1}(\lambda)\right)<\varepsilon / 4$ for all $\lambda \in P$. Note that

$$
d_{1}=d\left(\psi_{1}(P), C^{r}(K, N)-T_{\left(\left.f\right|_{M_{1}}\right)}^{l}\right)>0,
$$

since $\psi_{1}(P)$ is compact and $T_{\left(\left.f\right|_{M_{1}}\right)}^{l}$ is an open set.

Now we inductively construct continuous maps $\psi_{i}: P \rightarrow \mathcal{V}^{\prime}(i=2,3, \cdots)$ such that $\left.\psi_{i+1}\right|_{F}=\left.\psi_{i}\right|_{F}\left(=\left.\varphi\right|_{F}\right), \psi_{i+1}(P) \subset T_{\left(\left.f\right|_{M_{i+1}}\right)}^{l}$, and $d\left(\psi_{i}(\lambda), \psi_{i+1}(\lambda)\right)<$ $\min \left\{2^{-(i+2)} \varepsilon, 2^{-(i+1)} d_{1}, 2^{-i} d_{2}, \cdots, 2^{-2} d_{i}\right\}$ for all $\lambda \in P$, where

$$
d_{i}=d\left(\psi_{i}(P), C^{r}(K, N)-T_{\left(\left.f\right|_{M_{i}}\right)}^{l}\right)>0 .
$$

Then we define the map $\psi: P \rightarrow \overline{\mathcal{V}^{\prime}} \subset \mathcal{V}$ by

$$
\psi(\lambda)=\lim _{i \rightarrow \infty} \psi_{i}(\lambda)
$$

$(\lambda \in P)$. (Note that $\left\{\psi_{i}(\lambda)\right\}_{i=1}^{\infty}$ is a Cauchy sequence and that $C^{r}(K, N)$ is a complete metric space.) Then $\psi$ is a continuous map, $\left.\psi\right|_{F}=\left.\varphi\right|_{F}, \psi(P) \subset$ $\bigcap_{i=1}^{\infty} T_{\left(f \mid M_{i}\right)}^{l}=T_{f}^{l}$, and $d(\psi(\lambda), \varphi(\lambda)) \leq \varepsilon / 2<\varepsilon$ for all $\lambda \in P$. Choosing $\varepsilon$ smaller if necessary, we can show that $\varphi$ and $\psi$ are homotopic fixing $F$ by the same argument using $\pi: V(N) \rightarrow N$ as in the case where $f$ is proper. This completes the proof of Theorem 2.9 .

\section{Consequences about the homotopy groups}

Let $M, K$ and $N$ be $C^{r}$ manifolds of dimensions $m, k$ and $n$ respectively and $f: M \rightarrow N$ a $C^{r}$ map $(r \geq 1)$. In the following, we set $\mathcal{N}=C^{r}(K, N)$ and $\mathcal{R}_{f}^{q}=\mathcal{N}-T_{f}^{n-q-1}$. Note that for $q<n, \mathcal{R}_{f}^{q}$ is the union of a countable family of closed nowhere dense ${ }^{5}$ subsets of $\mathcal{N}$, provided that

$$
r \geq \frac{m+k-q}{n-q}
$$

\footnotetext{
${ }^{5} \mathrm{~A}$ subset $A$ of a space $X$ is nowhere dense if its closure $\bar{A}$ contains no nonempty open set.
} 
(See Theorem 2.4 and Remark 2.7.) Note also that when $K$ is a point, $\mathcal{N}$ and $\mathcal{R}_{f}^{q}$ are naturally identified with $N$ and $f\left(R_{q}(f)\right)$ respectively.

As a corollary to Theorem 2.9, we have the following.

Corollary 3.1. Let $M, K$ and $N$ be $C^{r}$ manifolds of dimensions $m, k$ and $n$ respectively and let $f: M \rightarrow N$ be a $C^{r}$ map $(r \geq 1)$. We suppose that $K$ is compact. Let $\mathcal{V}$ be an open subset of $\mathcal{N}$ which contains $T_{f}^{l}$. If

$$
r \geq \frac{m+k-n+l+1}{l-p+1} \quad \text { and } \quad p \leq l
$$

for a nonnegative integer $p$, then the homotopy group $\pi_{i}\left(\mathcal{V}, T_{f}^{l}\right)$ vanishes for all $i$ with $i \leq p$. In particular, $\pi_{i}\left(\mathcal{N}, T_{f}^{l}\right)$ vanishes for all $i$ with $i \leq p$. In other words, the inclusion $T_{f}^{l} \rightarrow \mathcal{N}$ induces an isomorphism $\pi_{i}\left(T_{f}^{l}\right) \rightarrow \pi_{i}(\mathcal{N})$ for all $i \leq p-1$ and an epimorphism for $i=p$ (i.e., the space $\left(\mathcal{N}, T_{f}^{l}\right)$ is p-connected).

Proof. Let $\varphi: D^{i} \rightarrow \mathcal{V}$ be an arbitrary continuous map such that $\varphi\left(\partial D^{i}\right) \subset T_{f}^{l}$. Then, since $i \leq p \leq l$ and

$$
r \geq \frac{m+k-n+l+1}{l-p+1} \geq \frac{m+k-n+l+1}{l-i+1}
$$

by Theorem 2.9 we see that $\varphi$ is homotopic to a map into $T_{f}^{l}$ by a homotopy fixing $\partial D^{i}$. Hence $\pi_{i}\left(\mathcal{V}, T_{f}^{l}\right)$ vanishes. This completes the proof.

Using the above corollary, we can obtain some results on the connectivity of the space $T_{f}^{l}$ as follows.

Example 3.2. Let $M$ and $K$ be $C^{r}$ manifolds of dimensions $m$ and $k$ respectively and let $f: M \rightarrow \mathbf{R}^{n}$ be a $C^{r}$ map $(r \geq 1)$. We suppose that $K$ is compact and that

$$
r \geq \frac{m+k-n+l+1}{l-p+1} \text { and } p \leq l
$$

for a nonnegative integer $p$. Then by the above corollary, $\pi_{i}\left(C^{r}\left(K, \mathbf{R}^{n}\right), T_{f}^{l}\right)$ vanishes for all $i$ with $i \leq p$. Since we have the exact sequence

$$
\pi_{i}\left(C^{r}\left(K, \mathbf{R}^{n}\right), T_{f}^{l}\right) \rightarrow \pi_{i-1}\left(T_{f}^{l}\right) \rightarrow \pi_{i-1}\left(C^{r}\left(K, \mathbf{R}^{n}\right)\right)
$$

and $\pi_{j}\left(C^{r}\left(K, \mathbf{R}^{n}\right)\right)$ vanishes for all $j$, we see that $\pi_{i}\left(T_{f}^{l}\right)$ vanishes for all $i$ with $i \leq p-1$. For example, putting $p=l=2$, we obtain that if $r \geq m+k-n+3$, then the space $T_{f}^{2}\left(\subset C^{r}\left(K, \mathbf{R}^{n}\right)\right)$ is 1-connected.

By an argument similar to the proof of Corollary 3.1, we can also prove the following ${ }^{6}$.

Corollary 3.3. Let $M, K$ and $N$ be $C^{r}$ manifolds of dimensions $m, k$ and $n$ respectively and let $f: M \rightarrow N$ be a $C^{r} \operatorname{map}(r \geq 1)$. We suppose that $K$ is compact. Let $\mathcal{V}$ be an open subset of $\mathcal{N}$ which contains $T_{f}^{l}$. If

$$
r \geq \frac{m+k-n+l+1}{l-p+1} \text { and } p \leq l
$$

for a nonnegative integer $p$, then the homology group $H_{i}\left(\mathcal{V}, T_{f}^{l} ; \mathbf{Z}\right)$ vanishes for all $i$ with $i \leq p$. In particular, $H_{i}\left(\mathcal{N}, T_{f}^{l} ; \mathbf{Z}\right)$ vanishes for all $i$ with $i \leq p$.

\footnotetext{
${ }^{6}$ We can also use the relative Hurewicz isomorphism theorem [Sp, p.397] together with the result of Corollary 3.1.
} 
In the above corollaries, if we consider the case where $K$ is a point, then $\mathcal{N}$ and $T_{f}^{l}$ are naturally identified with $N$ and $N-f\left(R_{q}(f)\right)$ with $q=n-l-1$ respectively. Thus we obtain the following.

Corollary 3.4. Let $M$ and $N$ be $C^{r}$ manifolds of dimensions $m$ and $n$ respectively and $f: M \rightarrow N$ a $C^{r}$ map $(r \geq 1)$. If

$$
r \geq \frac{m-q}{n-p-q} \quad \text { and } \quad n-q-1 \geq p,
$$

then the inclusion $N-f\left(R_{q}(f)\right) \rightarrow N$ induces an isomorphism $\pi_{i}\left(N-f\left(R_{q}(f)\right)\right) \rightarrow$ $\pi_{i}(N)$ for all $i \leq p-1$ and an epimorphism for $i=p$.

Corollary 3.5 (Church [C3]). Let $M$ and $N$ be $C^{r}$ manifolds of dimensions $m$ and $n$ respectively and $f: M \rightarrow N$ a $C^{r}$ map $(r \geq 1)$. Then the inclusion $N-$ $f\left(R_{q}(f)\right) \rightarrow N$ induces an isomorphism $\pi_{i}\left(N-f\left(R_{q}(f)\right)\right) \rightarrow \pi_{i}(N)$ for all $i$ with $i+q \leq n-2$ and $r \geq m-n+i+2$ and an epimorphism for all $i$ with $i+q \leq n-1$ and $r \geq m-n+i+1$.

Proof. First we consider the case where $i+q \leq n-2$ and $r \geq m-n+i+2$. In Theorem 2.9, put $p=i+1, k=0, l=n-q-1$. Then $p \leq l$ if and only if $i+1 \leq n-q-1$, which is satisfied by our assumption. Furthermore

$$
r \geq \frac{m+k-n+l+1}{l-p+1}
$$

if and only if

$$
r \geq \frac{m-q}{n-q-i-1}=1+\frac{m-n+i+1}{n-q-i-1} .
$$

When $m-n+i+1 \geq 0$, we have

$$
1+\frac{m-n+i+1}{n-q-i-1} \leq m-n+i+2 \leq r
$$

by our assumption. When $m-n+i+1<0$, we have

$$
\frac{m-q}{n-q-i-1}<1 \leq r .
$$

Thus we can apply Theorem 2.9 to see that the inclusion induces a monomorphism $\pi_{i}\left(N-f\left(R_{q}(f)\right)\right) \rightarrow \pi_{i}(N)$.

When $i+q \leq n-1$ and $r \geq m-n+i+1$, put $p=i, k=0$ and $l=n-q-1$, and we see that the inclusion induces an epimorphism $\pi_{i}\left(N-f\left(R_{q}(f)\right)\right) \rightarrow \pi_{i}(N)$ by a similar argument. This completes the proof.

By Corollary 3.3, we see that $H_{i}\left(\mathcal{N}-\mathcal{C}, \mathcal{N}-\mathcal{R}_{f}^{q} ; \mathbf{Z}\right)=0$ for all $i \leq n-q-s-1$ and for all $\mathcal{C} \subset \mathcal{R}_{f}^{q}$ with $\mathcal{C}$ closed in $\mathcal{N}$, provided that

$$
r \geq \frac{m+k-q}{s+1},
$$

where $q$ and $s$ are nonnegative integers. (For this, put $l=n-q-1$ and $s=l-p$.) Using this observation, we obtain the following.

Corollary 3.6. Let $M$ and $N$ be $C^{r}$ manifolds of dimensions $m$ and $n$ respectively and $f: M \rightarrow N$ a $C^{r}$ map $(r \geq 1)$. If 


$$
r \geq \frac{m-q}{s+1}
$$

for nonnegative integers $q$ and $s$, then $\operatorname{dim} f\left(R_{q}(f)\right) \leq q+s$.

Proof. First consider that case where $M$ is compact and hence $f$ is proper. Note that in this case $f\left(R_{q}(f)\right)$ is a compact subset of $N$. Without loss of generality, we may assume that $N$ is orientable. In the above observation, set $K$ to be a point. Then we see that $H_{i}\left(N-C, N-f\left(R_{q}(f)\right) ; \mathbf{Z}\right)=0$ for all $i \leq n-q-s-1$ and for all closed sets $C \subset f\left(R_{q}(f)\right)$. Since $H_{i}\left(N-C, N-f\left(R_{q}(f)\right)\right.$; $\left.\mathbf{Z}\right)$ is isomorphic to $\check{H}^{n-i}\left(f\left(R_{q}(f)\right), C ; \mathbf{Z}\right)$ (see [Sp, Theorem 10 (p.342)]), we see that the topological dimension of $f\left(R_{q}(f)\right)$ is at most $q+s$ (see [HW, Theorem VIII 4 (p.152)]).

When $M$ is not compact, it is a countable union of compact codimension 0 submanifolds. Thus $f\left(R_{q}(f)\right)$ is a countable union of closed sets whose topological dimensions are at most $q+s$. Then the conclusion follows from [HW, Theorem III 2 (p.30)]. This completes the proof.

Remark 3.7. In Corollary 3.6, the case where $s=0$ is nothing but [C3, Proposition 4] or [S3, Theorem 2 (p.173)]. To see that it is really a refinement, consider the following situation. Let $f: M \rightarrow N$ be a $C^{2}$ map between $C^{2}$ manifolds of dimension $m$. Then by Corollary 3.6, we see that $\operatorname{dim} f\left(R_{m-4}(f)\right) \leq m-3$. Note that this cannot be obtained directly from [C3, Proposition 4]. If, in addition, $R_{m-4}(f)=R_{m-2}(f)$ and $f$ is open and light ${ }^{7}$, then $\operatorname{dim} B_{f} \leq m-3$, where $B_{f}$ is the set of points in $M$ at which $f$ fails to be a local homeomorphism (see [C1]). Compare this with [C1, Corollary 1.5].

Remark 3.8. In [S3, §5], Sard conjectures that $f\left(R_{q}(f)\right)$ is of $(q+\rho)$-dimensional measure zero if $r \geq(m-q) / \rho$ for $\rho>0$. If this conjecture is true, putting $\rho=s+1$ for a nonnegative integer $s$, we obtain Corollary 3.6. In other words, Corollary 3.6 is a "supporting evidence" of the Sard conjecture.

The following is a refinement of Proposition 2.2.

Corollary 3.9. Let $f: M \rightarrow N$ be a $C^{r} \operatorname{map}(r \geq 1)$. If

$$
s \geq \max \left\{0, \frac{m-q-r}{r}\right\}
$$

for $q$ with $0 \leq q \leq \min \{m, n\}$, then $\operatorname{dim} f\left(R_{q}(f)\right) \leq q+s$.

Proof. We see that

$$
s \geq \frac{m-q-r}{r}
$$

if and only if

$$
r \geq \frac{m-q}{s+1} .
$$

Thus the result easily follows from Corollary 3.6. This completes the proof.

Definition 3.10. Let $\mathcal{A}$ be a subset of $\mathcal{N}$. For a nonnegative integer $t$, the codimension $\operatorname{codim} \mathcal{A}$ of $\mathcal{A}$ in $\mathcal{N}$ is greater than or equal to $t$ if for each continuous map $\varphi: P \rightarrow \mathcal{N}$ of a compact $p$-dimensional $C^{r}$ manifold $P$ with $p<t$, one can approximate $\varphi$ homotopically by another continuous map whose image does not intersect $\mathcal{A}$ and which is arbitrarily close to $\varphi$.

\footnotetext{
${ }^{7}$ A continuous map $f: M \rightarrow N$ is said to be light if, for every $y \in N, \operatorname{dim} f^{-1}(y) \leq 0$.
} 
In the proof of Theorem 2.9, if we put $F=\emptyset$, then $\psi$ is an approximation of $\varphi$ in the sense of Definition 3.10. Thus we obtain the following.

Corollary 3.11. Let $M, K$ and $N$ be $C^{r}$ manifolds of dimensions $m, k$ and $n$ respectively and let $f: M \rightarrow N$ be a $C^{r} \operatorname{map}(r \geq 1)$. We suppose that $K$ is compact. If

$$
r \geq \frac{m+k-n+l+1}{l-p+1} \text { and } \quad p \leq l
$$

then

$$
\operatorname{codim}\left(\mathcal{N}-T_{f}^{l}\right)>p
$$

Or equivalently

$$
\operatorname{codim}\left(\mathcal{R}_{f}^{q}\right) \geq \min \left\{n-q-1,\left[\frac{(r-1)(n-q)-(m+k-n)}{r}\right]\right\}+1,
$$

where $[a]$ denotes the integer part of $a \in \mathbf{Q}$.

Note that in the above corollary, when $r$ is sufficiently large, $\operatorname{codim}\left(\mathcal{R}_{f}^{q}\right) \geq n-q$.

\section{Added in Proof}

After the submission of the manuscript, the authors were informed that the Sard conjecture had already been affirmatively solved almost thirty years ago. See Theorem 3.4.3 of the book "Geometric measure theory" by Herbert Federer (Die Grundlehren der math. Wissenschaften, Band 153, Springer, Berlin, Heidelberg, New York, 1969). See also the historic backgrounds described on page 208 of the book. The authors would like to thank David Trotman, Patrice Orro and Georges Compte for having kindly informed them of this fact.

We also note that throughout the proof of Theorem 2.4, $r$ is assumed to be finite. However, a similar proof works also in the case where $r=\infty$ by virtue of the definition of the strong Whitney $C^{\infty}$-topology (for example, see page 36 of $[\mathrm{H}]$ ).

\section{REFERENCES}

[C1] P. T. Church, Differentiable open maps on manifolds, Trans. Amer. Math. Soc. 109 (1963), 87-100. MR 27:4245

[C2] P. T. Church, On points of Jacobian rank k, Trans. Amer. Math. Soc. 110 (1964), 413-423. MR 28:624

[C3] P. T. Church, On points of Jacobian rank k. II, Proc. Amer. Math. Soc. 16 (1965), 10351038. MR 31:6241

[DK] S. K. Donaldson and P. B. Kronheimer, The geometry of four manifolds, Oxford, 1990. MR 92a:57036

[GG] M. Golubitsky and V. Guillemin, Stable mappings and their singularities, Graduate Texts in Math. vol.74, Springer-Verlag, New York, Heidelberg, Berlin, 1973. MR 49:6269

[H] M. W. Hirsch, Differential topology, Graduate Texts in Math. vol.33, Springer-Verlag, New York, Heidelberg, Berlin, 1976. MR 96c:57001

[HW] W. Hurewicz and H. Wallman, Dimension theory, Princeton Univ. Press, Princeton, 1941. MR 3:312b

$[\mathrm{M}]$ J. Munkres, Obstructions to the smoothing of piecewise-differentiable homeomorphisms, Ann. of Math. 72 (1960), 521-554. MR 22:3005

[S1] A. Sard, The measure of the critical values of differentiable maps, Bull. Amer. Math. Soc. 48 (1942), 883-890. MR 4:153c

[S2] A. Sard, Images of critical sets, Ann. of Math. 68 (1958), 247-259. MR 20:6499

[S3] A. Sard, Hausdorff measure of critical images on Banach manifolds, Amer. J. Math. 87 (1965), 158-174. MR 30:3958 
[Sp] E. H. Spanier, Algebraic topology, McGraw-Hill, New York, San Francisco, St. Louis, Toronto, London, Sydney, 1966. MR 35:1007

[W] H. Whitney, Differentiable manifolds, Ann. of Math. 37 (1936), 645-680.

Departamento de Matemática, ICMSC-USP, Caixa Postal 668, 13560-970, São Carlos, SP, BRAZIL

E-mail address: biasi@icmsc.sc.usp.br

Department of Mathematics, Faculty of Science, Hiroshima University, HigashiHiroshima 739, JAPAN

E-mail address: saeki@top2.math.sci.hiroshima-u.ac.jp 